\title{
Space Software Defined Radio Characterization to Enable Reuse
}

\author{
Dale J. Mortensen ${ }^{1}$ and Daniel W. Bishop ${ }^{2}$ \\ Vantage Partners, LLC, Cleveland, Ohio, 44142 \\ David Chelmins ${ }^{3}$ \\ NASA Glenn Research Center, Cleveland, OH 44135
}

\begin{abstract}
NASA's Space Communication and Navigation Testbed is beginning operations on the International Space Station this year. The objective is to promote new software defined radio technologies and associated software application reuse, enabled by this first flight of NASA's Space Telecommunications Radio System architecture standard. The Space Station payload has three software defined radios onboard that allow for a wide variety of communications applications; however, each radio was only launched with one waveform application. By design the testbed allows new waveform applications to be uploaded and tested by experimenters in and outside of NASA. During the system integration phase of the testbed special waveform test modes and stand-alone test waveforms were used to characterize the SDR platforms for the future experiments. Characterization of the Testbed's JPL SDR using test waveforms and specialized ground test modes is discussed in this paper. One of the test waveforms, a record and playback application, can be utilized in a variety of ways, including new satellite on-orbit checkout as well as independent on-board testbed experiments.
\end{abstract}

\section{Introduction}

$\mathrm{T}$ HE National Aeronautics and Space Administration (NASA) is developing an on-orbit, adaptable, Software Defined Radio (SDR)/Space Telecommunications Radio System (STRS)-based testbed facility to conduct a suite of experiments to advance technologies, reduce risk, and enable future mission capabilities on the International Space Station (ISS). The Space Communications and Navigation (SCaN) Testbed Project will provide NASA, industry, other Government agencies, and academic partners the opportunity to develop and field communications, navigation, and networking technologies in the laboratory and space environment based on reconfigurable, SDR platforms and the STRS Architecture. Led by the NASA Glenn Research Center (GRC), the SCaN Testbed was developed to move SDR space technology forward, as the advancements of Appendix Table 2 indicate. The project was previously known as the Communications, Navigation, and Networking reConfigurable Testbed (CoNNeCT).

\section{A. Space Telecommunications Radio System (STRS)}

The STRS architecture is becoming a standard across NASA as software defined radios (SDRs) become more common for missions. Radio application portability between SDR platforms and reusability among missions are major objectives. The STRS architecture emphasizes platform abstraction and standard interfaces to aid portability and reuse.[1] Figure 1 illustrates how the STRS Operating Environment (OE) abstracts the SDR platform hardware and presents the common interface for all STRS applications.

\footnotetext{
${ }^{1}$ Waveform Developer, SCaN Testbed, 3000 Aerospace Pkwy., Brook Park, OH, non-Member.

${ }^{2}$ Waveform Developer, SCaN Testbed, 3000 Aerospace Pkwy., Brook Park, OH, non-Member.

${ }^{3}$ Experiments Lead, SCaN Testbed, 21000 Brookpark Rd, M.S. 54-8, non-Member.
} 
A STRS waveform repository is being developed to capture knowledge and artifacts, including code, models, and documentation, to facilitate application porting and reuse. The SCaN Testbed's launch waveforms and subsequent experiments will comprise the first entries.

\section{B. SCaN Testbed Overview}

The SCAN Testbed has three SDRs. In addition to the capabilities identified below, all radios are capable of supporting networking, navigation, time transfer, file management, and data analysis.

- The Jet Propulsion Lab (JPL) SDR leverages off the developments of the Electra radio. This SDR is

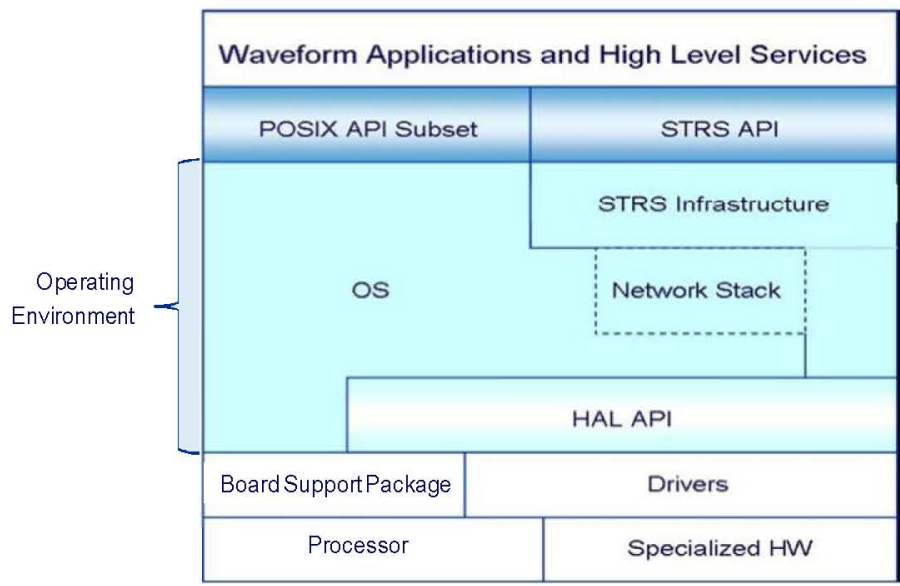

Figure 1. STRS abstraction layering capable of full-duplex, Tracking and Data Relay Satellite System (TDRSS)-compatible, STRS-compliant S-band communications and receive-only GPS L-band navigation. The S-Band and GPS can be operated simultaneously.

- The Harris Corporation SDR was developed under a cooperative agreement with GRC. This SDR is capable of full-duplex, TDRSS-compatible, STRS-compliant Ka-band communications.

- The General Dynamics (GD) SDR was developed under a cooperative agreement with GRC. This radio leverages developments of the TDRSS fourth-generation transponder and is capable of full-duplex, TDRSS-compatible, STRS-compliant S-band communications.

The radios are mounted to the Flight Enclosure and functionally interface with the Avionics and RF systems as shown in Fig. 2. Note certain configurations enable multiple SDR use for networking and/or routing experiments. Table 3 in the Appendix gives more detailed specifications for the SDRs. Additional information about the SCaN Testbed can be found at: http://spaceflightsystems. grc.nasa.gov/SOPO/SCO/SCaNTestbed/

\section{Avionics}

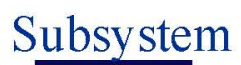

SDR

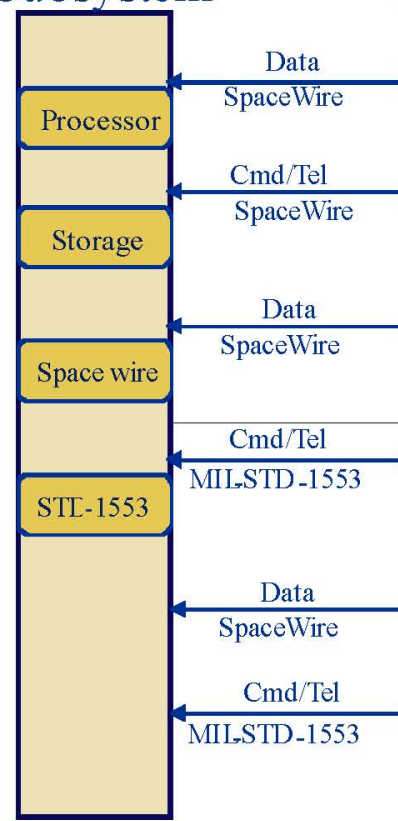

GD SDR

JPL SDR

\section{Subsystem

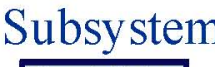

RF

Subsystem
Antenna Subsystem
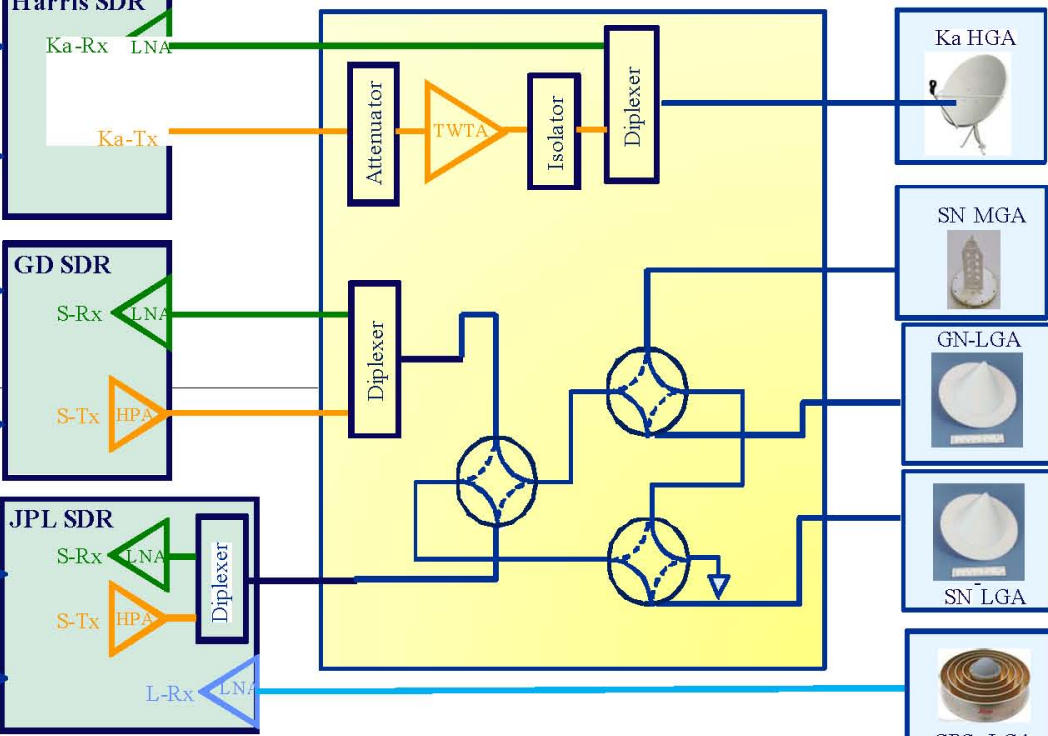

Figure 2. SCaN Testbed System Diagram 
Table 1. Launch Operational Waveforms Summary

\begin{tabular}{|c|l|l|c|c|}
\hline SDR & \multicolumn{1}{|c|}{ TDRSS Mode } & \multicolumn{1}{|c|}{ Modulation } & $\begin{array}{c}\text { Tx Data } \\
\text { Rate } \\
(\mathrm{kbps})\end{array}$ & $\begin{array}{c}\text { Rx Data } \\
\text { Rate } \\
(\mathrm{kbps})\end{array}$ \\
\hline GD & S-band DG1, Mode 1 & SQPN I/Q Power Ratio - 1:1 & 192 & 72 \\
\hline GD & S-band DG1, Mode 3 & QPSK I/Q power ratio - 1:4. & $\begin{array}{c}\text { Q:1000 } \\
\text { I: 1 }\end{array}$ & 72 \\
\hline GD & S-band DG2 & SQPSK I/Q power ratio 1:1 & 1000 & 72 \\
\hline JPL & S-bandDG1, Mode 2 & SS-BPSK & 24 & 18 \\
\hline JPL & S-band DG2 & BPSK & 769 & 769 \\
\hline Harris & Ka-band DG2 & SQPSK I/Q power ratio 1:1 & 100000 & 12500 \\
\hline
\end{tabular}

\section{Launch Waveforms Overview}

There is an operational STRS-compliant waveform application on each of the Testbed's SDRs at launch. These baseline waveforms offer Space Network and some Near Earth Network connectivity, as listed in Table 1, for experimenters. These waveforms have been run through a rigor of tests to ensure operation in space with the intended service. Modifications to add capability to these existing waveforms are already planned as the first set of experiments. There is a high-fidelity engineering model of the SCaN Testbed, known as the ground integration unit, that is used for experiment development and testing prior to uploading a new waveform.

The remainder of the paper will primarily focus on the JPL SDR characterization using the baseline operational waveform named the GGT. Separate test waveforms for the JPL SDR are also discussed. The GGT Waveform is unique in a couple of ways. It was the first STRS waveform ported from one SDR to another, enabled by the STRS OE's common application programmers interface. [2] Secondly, it was the first operational waveform integrated with the JPL SDR, and so the effort included some radio characterization, mainly related to temperature compensation and optimization.

\section{Temperature Compensation}

Several of the hardware components in the JPL SDR's RF module have performance parameters that vary over the wide space environment temperature range. For TDRSS compatibility, the absolute frequency needs to be within $+/-700 \mathrm{~Hz}$ of the designated carrier. Since the oscillator source does not maintain that level of accuracy over the temperature range, a software based trim is done by an appropriate offset in the baseband waveform. The frequency offset based on temperature was characterized during environmental thermal vacuum testing. The calibration data was integrated as a look-up table in the launch waveform and this data is available for subsequent experimental waveforms.

Temperature changes affect the IQ modulator of the RF module in more complex ways. Analog modulators' performance can benefit from digital baseband pre-compensation to mitigate undesired effects, especially those that vary over the operational temperature range. The effect, if not corrected, will negatively affect the Error Vector Magnitude (EVM) and subsequently the Bit Error Rate (BER). The baseline waveform was designed with specific STRS ground test modes to characterize the IQ Modulator with DC offset, level, and phase sweeps. These modes will be described in the ground test subsection of Waveform Test Modes (IV-A).

In order to compensate for modulator-induced imbalance, the I and Q digital words are adjusted. The adjusted values are sent to the digital-to-analog converter (DAC), which produces adjusted voltages that are passed to the modulator. The modulator adds temperature-dependent error, which then results in a near-ideal constellation if the adjustment process has been done correctly. The mathematical representation of the adjustments is:

$$
\begin{aligned}
& I^{\prime}(t)=\alpha_{\mathrm{I}} \cdot \cos (\omega t)+\beta_{I} \\
& Q^{\prime}(t)=\alpha_{\mathrm{Q}} \cdot \sin (\omega t+\theta)+\beta_{Q}
\end{aligned}
$$

There are a total of 5 adjustment parameters. The $\alpha$ parameters are the I and Q levels. The $\beta$ parameters are the I and Q DC offsets. The $\theta$ parameter is the Q phase offset. The I and Q levels set the scale factor of the waveform sent 
to the modulator. The I and Q DC offsets are simply an adjustment that causes the sine and cosine to oscillate around a value other than 0 . The Q phase offset is a modification to the standard 90-degree difference between the I and $Q$ values sent to the modulator.

\section{Platform Test Waveforms}

A test waveform serves a specific non-operational purpose in the SDR application world. Test waveforms are often used by SDR platform developers for verifying hardware operations and characterizing performance. The SCaN Testbed's JPL SDR has some very useful test waveforms in its launch configuration: S-band capture, S-band playback, and GPS capture. These are available to aid experimenters in developing their own waveforms on the ground, as well as being a part of experiment execution on-orbit.

\section{A. S-band Capture}

Having a generic S-band capture application is an important development tool for the radio hardware and software. Initially this test waveform served to verify functionality of the SDR and integrated OE. During SCaN Testbed system integration the S-band capture waveform was used to debug an analog-to-digital converter (ADC) sample clocking problem and perform testing of the system noise figure.

The S-band capture waveform supported a test of the system noise figure. An external noise generator unit produces noise at a calibrated Excess Noise Ratio (ENR) for a given frequency. The ENR provides an increase in the effective noise temperature of the RF path with respect to ambient. The S-band capture technique was used to record raw values from the ADC with the noise source on then off, and the automatic gain control (AGC) set to various duty cycles. Analysis of this dataset using the Y-method allowed computation of a system noise figure within $0.3 \mathrm{~dB}$ of that measured using external test equipment.[3] A similar technique could later be applied on-orbit to calibrate the receiver gain variation over time given the large difference between noise temperature for space- and Earth-facing antennas.

The S-band capture technique is a critical tool for system-level debugging. Capturing the current RF state of an antenna allows identification of interferers in the overall RF environment through simple post-processing. This makes it easier to diagnose external transceiver performance issues, while ruling out hardware causes. With the SDR's on-board RAM, over one second of the received signal can be captured. A software application can control a trigger for the capture based on level or other experiment parameters.

\section{B. S-band Playback}

Similarly the S-band playback test waveform uses the on-board RAM to store DAC samples for transmission. There is memory capacity on the JPL SDR for over half a second worth of playback, which can be repeated for continuous transmission. Being able to transmit any sequence of samples makes the S-band playback test waveform very versatile. During $\mathrm{SCaN}$ Testbed integration it was used to aide in calibration of the SDR over temperature. A 16QAM constellation was produced from which IQ imbalance could be measured and then compensated for in most lower order modulation schemes.

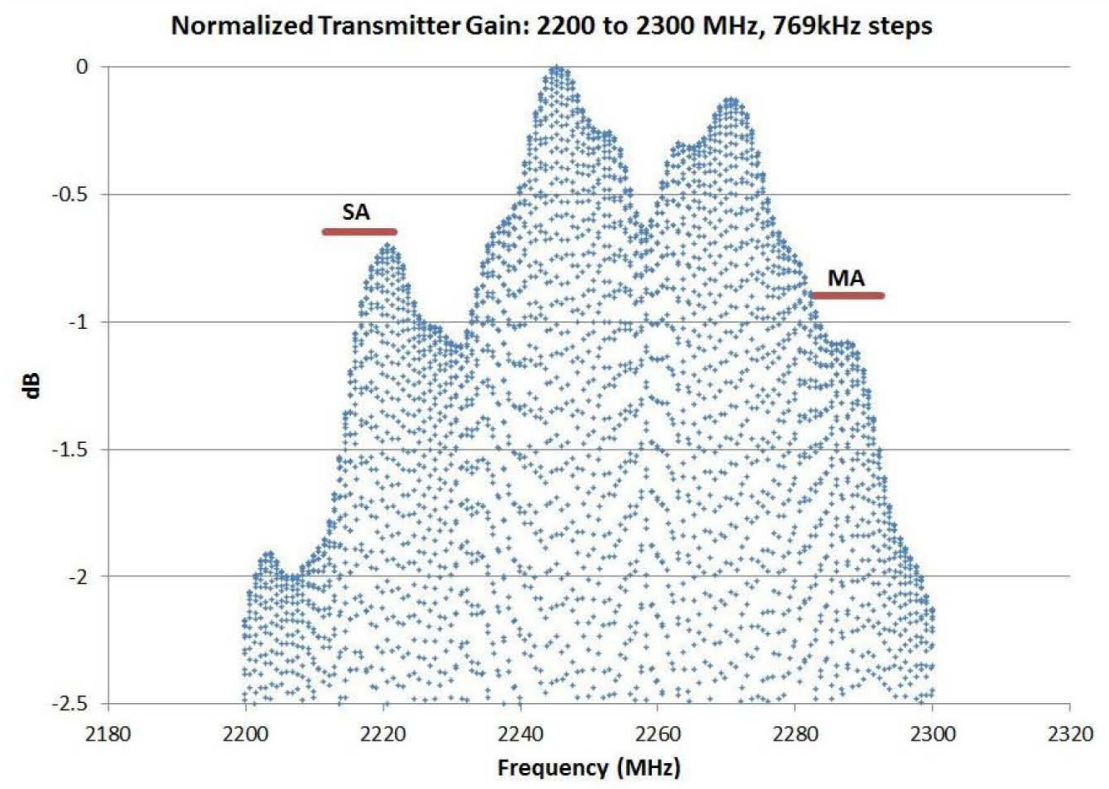

Figure 3. JPL SDR Output Flatness 
The S-band playback test waveform was successfully used in the characterization of the transmit RF passband. To sweep the entire S-band frequency range of the SDR the test waveform was used to generate a continuous wave (CW) while the upconverter synthesizer was stepped through the 2.2-2.3 GHz band. This was done to obtain the gain versus frequency characteristics, as shown in Fig. 3. The "SA" and "MA" markings indicate the two operational frequencies of the launch waveform.

\section{GPS Capture}

The GPS capture test waveform has been used to verify functionality of the L-band receiver hardware throughout system integration. It has been a critical test waveform since there is no operational real-time GPS waveform currently on the SDR. Data files captured from the test waveform are downloaded and post-processed to observe GPS or other input test signals. The operational waveform to calculate position, velocity, and time data from the GPS signal is being developed with plans for upload and testing in 2013. This may be available for additional experiments in the GPS domain. One of the key capabilities of the SCaN Testbed is the L5 frequency capability, which allows evaluation of experimental waveforms at this new frequency.

The design of the GPS capture waveform is similar in nature to the S-band capture except the data source is from the GPS Module instead of the RF Module. Received RF is filtered through separate L1, L2, and L5 hardware chains and provided to the Baseband Processor Module as several simultaneous data streams.

\section{Waveform Test Modes}

\section{A. Ground Test}

As defined by STRS, a ground test is a waveform function intended for development, integration, or environmental testing purposes usually conducted on the ground before deployment. The testing may include calibration (e.g., temperature compensation), fault detection and isolation (e.g., sequential triggering of the waveform processing chain), or debugging capabilities (e.g., logging of instantaneous radio configuration). The STRS ground test is the software analogy to the hardware ground support equipment (GSE) port, only available on the ground during pre-launch testing.

The GGT Waveform employs STRS ground test features for calibration with the SDR platform during prelaunch testing. Six ground test modes were created to sweep the IQ modulator parameters so as to optimize IQ modulator performance. This was done at several points over a wide temperature range, with separate ground test modes to perform sweeps on the 5 adjustment parameters described in section II. The sixth ground test performs a sweep of the scale factor for I and Q levels. This allows evaluation of the integrated power amplifier compression curve.

\section{B. Run Test}

The STRS architecture allows for both waveform groundtest and runtest commands, which provide special capabilities for waveform designers and operators. As implied by its name, a ground test is distinct in that the command is removed from the operational waveform software build, while a run test remains a part of the waveform during operations.

The GGT Waveform includes 7 run tests, all of which return instantaneous state information for the waveform and/or hardware. The simplest run tests return waveform state (running or stopped) and version information for the waveform and device drivers. There is a run test to return values for all of the FPGA registers, which allows realtime debugging of operational algorithms, as well as a run test that forces the waveform to verify that all FPGA registers are properly set. A fifth run test reports the cumulative bit error rate for the waveform when operating in bit error rate test mode. The remaining two run tests provide information on the temperature compensation table values and the automatic level control settings for each waveform mode.

Although the GGT implementation of the runtest method provides mainly health and status information, the STRS architecture does not restrict functionality. A run test can also be a powerful tool for manipulating waveform characteristics. The transmitter frequency, power level, and spectrum can all be changed on-the-fly to accommodate system design testing. For example, profiles can be generated to perform frequency shifts while simultaneously varying amplitude on an existing waveform. The SCaN Testbed can provide this function to allow self-checkout as well as checkout of other communication assets. 


\section{Test Waveforms On-Orbit}

On-orbit characterization of the SCaN Testbed may also employ test waveforms. The SCaN Testbed Project has several NASA proposed and industry proposed experimental waveforms that can be used to characterize operational links. Many space links operate with minimal link margin so it is imperative to ensure the equipment is running as close to optimal as possible. Correspondingly if the links do not close then having a few test tools can be instrumental in determining cause(s) for the reduced performance. The JPL SDR has the S-band Capture/Playback and GPS Capture Test Waveforms described previously. These applications can be building blocks for more sophisticated test waveforms, such as for signal and spectral analysis.

\section{A. RF Loopback and Transponding}

There are sometimes cases where the memory is not deep enough to store a full event of interest or the experimenter wants to be able to look at things in real-time. In these cases a loopback waveform can be very useful. Since each SDR platform was characterized the response of the radio receivers is known, and so the response of a sending spacecraft and the channel can be found. The measurement could occur in real-time so that things such as interferers and fading could be observed.

\section{B. FFT and raw ADC samples}

Spectral analysis is a basic tool that can be used in many ways to investigate potential issues with a transmitter. The SDR allows a large flexibility on the parameters for the FFT to give the experimenter the ability to extract anomalies of the source transmitter. The experimenter would be able to verify the transmitter's bandwidth, spectral shape, and frequency and this could all be done in real-time. The real-time FFT could also help diagnose random noise or interferers that could be affecting a link. The real-time FFT could be coupled with a level trigger and memory which could diagnose power on and off issues of a transmitter.

\section{Conversion of Ground Tests}

A STRS run test requires a specific and proper end, as well as a returned status based upon test. For the GGT Waveform the ground test code is not part of the flight software build, but the code could be rewritten to become run tests if appropriate in an experiment. For example, the frequency sweep ground test as a run test could be used to generate a Doppler-like sweep to test another receiver's ability to track a shifting frequency. Or as a run test the transmit level sweep could be modified to check loss of signal, acquisition of signal, or to find the operating range of another platform.

\section{Conclusion}

The SCaN Testbed has been characterized enabling an experimenter to distinguish testbed contributions versus characteristics of the link, source radio, or interferer. The SCaN Testbed offers a unique orbiting platform that can be utilized in a variety of ways for new waveform experiments or to characterize other platforms. The testbed is configurable so it can operate with a number of other spacecraft, satellite, and ground services. The launched operational and test waveforms already allow experimenters much flexibility. On the JPL SDR there are the S-band capture and playback, as well as the GPS capture test waveforms. These applications coupled with the radio's memory storage can be building blocks for an experiment. The on-orbit testbed as well as the ground integration unit are designed specifically for new waveforms to be developed, uploaded, and operated. The baseline operational and test waveforms already launched are the beginning of the STRS waveform application repository, integral to the $\mathrm{SCaN}$ Testbed experiment program, and a resource for future missions. 


\section{Appendix}

Table 2. SCaN Testbed Advancements

\begin{tabular}{|c|c|c|}
\hline $\begin{array}{l}\text { STRS/SDR } \\
\text { Development }\end{array}$ & $\begin{array}{l}\text { STRS compliant SDRs for commonality across different } \\
\text { developers }\end{array}$ & $\begin{array}{l}\text { Unique proprietary SDR } \\
\text { architectures }\end{array}$ \\
\hline $\begin{array}{l}\text { SDR TRL } \\
\text { Advancement }\end{array}$ & $\begin{array}{l}\text { Advance } 3 \text { SDR platforms (S-band, Ka-band) to TRL-7 } \\
\text { for future NASA applications } \\
\text { Faster processor, Higher V2 FPGA density \& V4 }\end{array}$ & $\begin{array}{l}\text { UHF Electra (MRO, MSL) } \\
\text { Mini-RF (LRO) }\end{array}$ \\
\hline $\begin{array}{l}\text { In-flight SDR } \\
\text { Reconfiguration }\end{array}$ & Routine in-orbit SDR reconfiguration & $\begin{array}{l}\text { Limited to essential } \\
\text { reconfigurations and demos }\end{array}$ \\
\hline $\begin{array}{l}\text { S-band } \\
\text { Communications }\end{array}$ & $\begin{array}{l}\text { STRS-compliant, firmware based waveform } \\
\text { (S-band } \sim 10 \mathrm{MHz} \text { class, } \sim 8 \mathrm{Mbps} \text { QPSK) }\end{array}$ & Fixed TDRSS IV transponder \\
\hline $\begin{array}{l}\text { Ka-band } \\
\text { Communications }\end{array}$ & $\begin{array}{l}1^{\text {st }} \text { NASA TDRSS Ka-band user } \\
(200 \mathrm{MHz}, 100 \mathrm{Mbps}, \text { SQPSK) }\end{array}$ & $\begin{array}{l}\text { LRO, SDO Ka-band direct to } \\
\text { Earth }\end{array}$ \\
\hline GPS Navigation & $\begin{array}{l}1^{\text {st }} \text { GPS L } 5 \text { space receiver } \\
\text { SDR-based GPS at L } 1, \text { L } 2 \text { c L } 5 \text { capability }\end{array}$ & $\begin{array}{l}\text { Blackjack L 1, L 2c } \\
\text { Fixed capability }\end{array}$ \\
\hline $\begin{array}{l}\text { Networking/ } \\
\text { Routing }\end{array}$ & $\begin{array}{l}\text { DTN (routing of bundles among multiple SDRs and } \\
\text { S/Ka-band links.) } \\
\text { DTN in SDR ("Radio As a Node") provides cross-layer } \\
\text { optimization incorporating RF link status. } \\
\text { Routing and QoS-driven handling of DTN bundles in } \\
\text { response to changes in traffic and RF link quality }\end{array}$ & $\begin{array}{l}\text { CANDOS and CLEO } \\
\text { demonstrated IP in space and } \\
\text { conventional mobile IP } \\
\text { DTN demonstrations in flight } \\
\text { computer, separate from radio }\end{array}$ \\
\hline
\end{tabular}

Table 3. SDR Radio Specification

\begin{tabular}{|l|l|l|l|}
\hline & \multicolumn{1}{|c|}{ JPL Radio } & \multicolumn{1}{|c|}{ GD Radio } \\
\hline Processor & SPARC V8 & Coldfire Processor & \multicolumn{1}{c|}{ Harris Radio } \\
\hline Memory & SDRAM + Flash & $\begin{array}{l}\text { CRAM + SDRAM + } \\
\text { EEPROM }\end{array}$ & EDAC SDRAM \\
\hline FPGAs & 2x Xilinx Virtex II FPGAs & Xilinx Virtex II FPGA & 4x Xilinx Virtex IV FPGAs \\
\hline Additional & & & DSP (1 GFLOP) \\
\hline $\begin{array}{l}\text { Command } \\
\text { \&Data }\end{array}$ & $\begin{array}{l}\text { MIL-STD-1553B for command } \\
\text { \& telemetry }\end{array}$ & $\begin{array}{l}\text { MIL-STD-1553B for command } \\
\& \text { telemetry }\end{array}$ & $\begin{array}{l}\text { SpaceWire Link for data and } \\
\text { for command \& telemetry }\end{array}$ \\
\hline RF Link & Full Duplex S-band & Full Duplex S-band & Full Duplex Ka-band \\
\hline TX Specs & Tx:2.2-2.3 GHz, 5-10W output & Tx:2.2-2.3 GHz, 8W output & $\begin{array}{l}\text { Tx: 25.65-26.16 GHz, } \\
225 \mathrm{MHz} \text { BW }\end{array}$ \\
\hline RX Specs & $\begin{array}{l}\text { Rx: 2.025-2.12 GHz, } \\
11 \text { MHz BW }\end{array}$ & $\begin{array}{l}\text { Rx: 2.025-2.12 GHz, } \\
6 \text { MHz BW }\end{array}$ & $\begin{array}{l}\text { Rx: 22.5-23.1 GHz, } \\
50 \text { MHz BW }\end{array}$ \\
\hline GPS & $\begin{array}{l}\text { GPS Receive Sampler: } \\
\text { L1,L2, and L5 }\end{array}$ & & \\
\hline RTOS & RTEMS & VxWorks & VxWorks \\
\hline
\end{tabular}




\section{Acknowledgments}

The authors would like to recognize the JPL Team of Jim Lux, Greg Taylor, Ken Peters, and support staff for developing the S-band capture/playback and GPS capture test waveforms for the SCaN Testbed JPL SDR.

\section{References}

[1]"Space Telecommunications Radio System (STRS) Architecture Standard Release 1.02.1". NASA Technical Memorandum 2010-216809, December 2010. Available online at the NASA Technical Reports Server.

[2] Mortensen, D.J., “'STRS Waveform Porting for NASA's CoNNeCT Project”. SDR'11 - Wireless Innovation Conference, Session 7B: Government Applications, December 2, 2011.

[3] "Noise Figure Measurement Accuracy - The Y-Factor Method", Agilent Application Note 57-2. 